\title{
SOC estimation of Lithium-ion battery based on Kalman filter algorithm
}

\author{
Yu Ding-xuan \\ School of Mechatronic Engineering and Automation \\ Shanghai University \\ Shanghai 200072,China \\ edmend_yu@163.com
}

\author{
Gao Yan-xia \\ School of Mechatronic Engineering and Automation \\ Shanghai University \\ Shanghai 200072, China \\ gaoyanxia@shu.edu.cn
}

\begin{abstract}
This paper presents Extended Kalman-filter (EKF) algorithm which is based on a first-order Lithium-ion batteries model. Curve fitting According to the OCV(open circuit voltage)、SOC(state of charge) parameters measured in experiments, descript status equation and observation equation of Lithium-ion battery in detail , so that it can accurately demonstrates the characteristics of the Lithium-ion battery. Simulation and experiment results show the feasibility and effectiveness of the algorithm.
\end{abstract}

\section{Keywords-Kalman filter; Lithium-ion battery; SOC}

\section{INTRODUCTION}

With the advantages of energy-saving and friendly to the environment, Hybrid Electric Vehicle and pure Electric vehicle have been developing rapidly. Proper operation of these new type vehicles rely on batteries and their manage system; therefore the stability and reliability of power batteries are important for energy transformation ${ }^{[1]}$.

One of the most important parameter in battery management system is state of charge (SOC). State of charge means the proportion of released energy from all battery energy storage. Only SOC estimated precisely, energy balance can be done without damaging the batteries. However due to battery complex internal chemical and physical reaction, SOC cannot be measured directly and multiple factors should be considered, so it is difficult to estimate SOC when battery is working.

Ampere-hour (Ah) method is the most commonly used estimation method. However integral calculation has accumulation error, with the growth of working time, accumulation error will result in SOC estimation error. Ampere-hour method is suitable for full charge and full discharge situation, but not for hybrid electric vehicles. Besides Ampere-hour method, other normally used SOC estimation method includes open circuit voltage method, neural network method. Open circuit voltage is often used in battery overhaul rather than state of charge estimation; neural network models are too complicated, and in order to train the model, plenty of data are needed ${ }^{[2]}$.

The method presented in this paper is extended algorithm based on Kalman filter. The core of extended Kalman filter algorithm is to propose a precise state describe model, according to the measurement data to calculate the values of objects with recursive algorithm. Comparing to other algorithms, extended Kalman filter algorithm has its own advantages: even if there were errors in system, self-correction can be down within a certain range and op- timal estimation value can be obtained. Kalman filter algorithm needs accurate state describe model and accurate measurement values, beyond all of these, EKF has no request on initial value.

\section{THE BATTERY MODEL}

The purpose of building battery model is to establish battery internal state variables, external quantitative relation electrical characteristics and to set up the mathematical model, then based on the external variables such as battery voltage, current, temperature to calculate internal state variables like SOC, internal resistance and electromotive force.

There are many battery models, in order to guarantee the precision, in this article I chooses Thevenin model as Lithium battery model. This kind of model has good dynamic performance and can simulate the dynamic characteristics of Lithium ion battery accurately ${ }^{[3]}$.

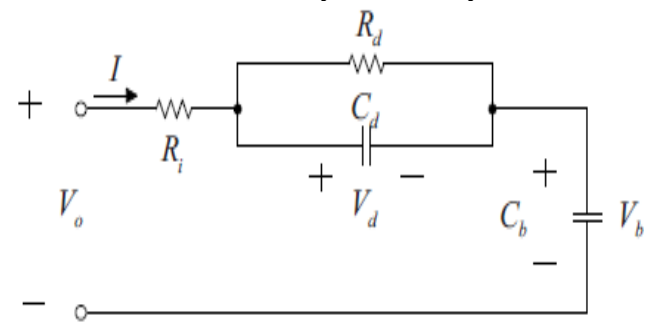

Fig.1 Thevenin battery model

In figure.2, I represent battery charging and discharging current flow; $\mathrm{V}_{0}$ represent battery terminal voltage. Thevenin dynamic circuit model includes: 1.the battery open circuit voltage $V_{d}$; 2.the battery internal resistance $R$, including ohm resistance $R_{i}$ and electrochemical polarization resistance $R_{d}$; 3.equivalent capacitance $C_{d}$ is to describe cell electrochemical polarization and concentration polarization, which reflects the battery transient response of charge or discharge. According to the Kirchhoff's voltage and current laws, and capacitance voltage variation and its current relationship, Thevenin circuit model of the state space equation can be expressed as ${ }^{[4]}$ :

$$
\begin{gathered}
\dot{V}_{b}=\frac{I}{C_{b}} . \\
\dot{V}_{d}=-\frac{I}{R_{d} C_{d}} V_{d}+\frac{1}{C_{d}} I . \\
V_{0}=V_{b}+V_{d}+I R_{i} .
\end{gathered}
$$

The relationship of OCV and SOC for Lithium ion battery is not linear. Drawing the curve graph base on the 
measurement data, an OCV function relation about SOC can be obtained:

$$
V_{b}=k * S O C+d .
$$

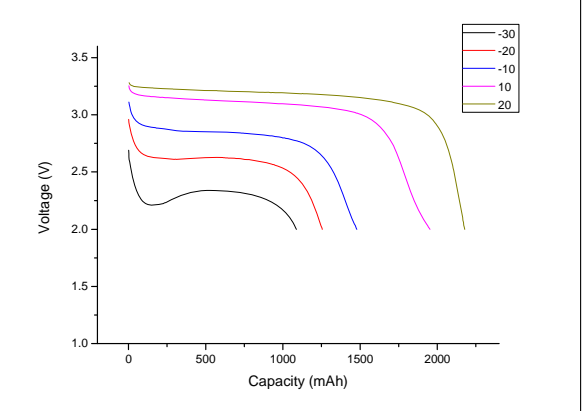

Fig.2 OCV and SOC curve from $-30 \mathrm{C} \cdot \sim 20 \mathrm{C}$.

Based on the equations above, the system equations can be derived:

$$
\begin{gathered}
{\left[\begin{array}{c}
S O C \\
V_{d}
\end{array}\right]=\left[\begin{array}{c}
\frac{I}{k C_{b}} \\
-\frac{I}{R_{d} C_{d}} V_{d}+\frac{1}{C_{d}} I
\end{array}\right]} \\
V_{0}=k S O C+V_{d}+I R_{i}+d .
\end{gathered}
$$

These two equations are state equations and observation equation for Lithium ion battery model, discretization for continuous equation and SOC calculation will be done in next part.

\section{THE EKF ESTIMATION PRINCIPLE OF SOC}

Kalman filtering method algorithm equation can be divided into two parts [5]:

1. Predict system status, system output and error;

2. Correct the current state estimate value based on system output value.

When the system's state equation is nonlinear, Kalman filtering method cannot be put into use directly, system equation discretization is required, and this kind of nonlinear equation for discretization of the Kalman filtering method is called extended Kalman filtering method (EKF). According to equations (5) (6), the system state equation can be written as:

$$
\boldsymbol{x}=\left[\begin{array}{ll}
x_{1} & x_{2}
\end{array}\right]^{T}, x_{1}(t)=S O C, x_{2}(t)=V_{d} .
$$

System input is defined as $u(t)=I$, output is defined as $y(t)=V 0$, then the battery model can be written as:

$$
\begin{aligned}
\dot{\boldsymbol{x}} & =f(\boldsymbol{x}, \boldsymbol{u})+\boldsymbol{w} . \\
\dot{\boldsymbol{y}} & =g(\boldsymbol{x}, \boldsymbol{u})+\boldsymbol{v} .
\end{aligned}
$$

Among these,

$$
\begin{gathered}
f(x, u)=\left[\begin{array}{c}
\frac{u}{k C b} \\
-\frac{I}{R_{d} C_{d}} x_{2}+\frac{1}{C_{d}} u
\end{array}\right] . \\
g(x, u)=k x_{1}+x_{2}+R_{i} u+d .
\end{gathered}
$$

In these formulas, $\mathrm{w}$ and $\mathrm{v}$ represent system noise and measurement noise. For formulas (8) (9), Taylor series ex- pansion is needed for the linearization, the model after linearization is:

$$
\begin{aligned}
\dot{x} & =A_{t} \cdot x+B_{t} \cdot u+w \\
\dot{y} & =C_{t} \cdot x+D_{t} \cdot u+v .
\end{aligned}
$$

Among these,

$$
\begin{aligned}
& \boldsymbol{A}_{t}=\frac{\partial f(\boldsymbol{x}, \boldsymbol{u})}{\partial \boldsymbol{x}} \mid x(t), u(t)=\left[\begin{array}{cc}
0 & 0 \\
0 & -\frac{I}{R_{d} C_{d}}
\end{array}\right] \\
& \boldsymbol{B}_{\boldsymbol{t}}=\frac{\partial f(\boldsymbol{x}, \boldsymbol{u})}{\partial \boldsymbol{u}} \mid x(t), u(t)=\left[\begin{array}{c}
\frac{1}{k C_{b}} \\
\frac{1}{C_{d}}
\end{array}\right] . \\
& \boldsymbol{C}_{t}=\frac{\partial g(\boldsymbol{x}, \boldsymbol{u})}{\partial \boldsymbol{x}} \mid x(t), u(t)=\left[\begin{array}{ll}
k & 1
\end{array}\right] \text {. } \\
& \boldsymbol{D}_{\boldsymbol{t}}=\frac{\partial g(\boldsymbol{x}, \boldsymbol{u})}{\partial \boldsymbol{u}} \mid x(t), u(t)=R_{i} .
\end{aligned}
$$

After discretization, the model can be expressed as:

$$
\begin{aligned}
& x_{k}+1=A_{k} \cdot X_{k}+B_{k} \cdot U_{k}+w_{k} . \\
& y_{k}=C_{k} \cdot X_{k}+D_{k} \cdot U_{k}+v_{k} .
\end{aligned}
$$

Extended Kalman filtering algorithm calculation steps are as follows:

Initialization: $\mathrm{k}=0$, Set initial condition $\mathrm{x}^{*} \mathrm{k}$, Error covariance matrix $\mathrm{P}^{*}$ and noise covariance matrix $\mathrm{Q}^{*}$ 。

calculation : $k=1,2,3 \ldots \ldots$.

update state estimation :

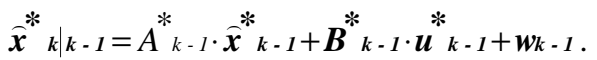

update error covariance :

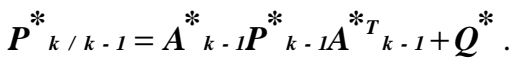

Kalman gain matrix :

$$
L k=P_{k / k-1}^{*} C_{k}^{* T}\left(C_{k}^{*} P_{k / k-1}^{*} C_{k}^{*}\right)^{-1} \text {. }
$$

update state estimation and measurement results :

$$
\widehat{\boldsymbol{X}}_{k}^{*}=\widehat{\boldsymbol{X}}_{k \mid k-1}^{*}+\boldsymbol{L} k\left[\boldsymbol{y}_{k}^{*}-\left(C_{k}^{*}{ }_{\boldsymbol{X}}^{*}{ }_{k \mid k-1}+D_{k \boldsymbol{k}}^{*}+\boldsymbol{v}\right)\right] .(19)
$$

update results of error covariance :

$$
\boldsymbol{P}^{*}{ }_{k}=\left(I-L_{k} C^{*}{ }_{k}\right) P^{*}{ }_{k / k-1} \text {. }
$$

Extended Kalman filtering method include above five basic formulas, which can calculate the system state after put parameters into the formulas. The core of EKF was to compare the predicted value and the measurement value, adjusting Kalman gain according to the size of the error, the gain will be used to calculate the next predicted value. The bigger the error, the greater the gain, the bigger the estimated value will be corrected; the lesser the error, the smaller the gain, the lesser the estimated value will be corrected. Based on such a thought, extended Kalman filtering method has good real time calculation performance ${ }^{[6]}$. 


\section{THE SIMULATION AND EXPERIMENT}

\section{A . The experiment system}

In order to validate the result of extended Kalman filtering algorithm, the simulation results were compared with the experimental data in the Matlab simulation. The architecture of a system diagram showed below, In order to obtain the parameters for calculation, it contains a current measurement circuit, voltage measurement circuit and low-pass filter circuit which is used to filter out signal noise $\mathrm{e}^{[\mathrm{7]}}$ :

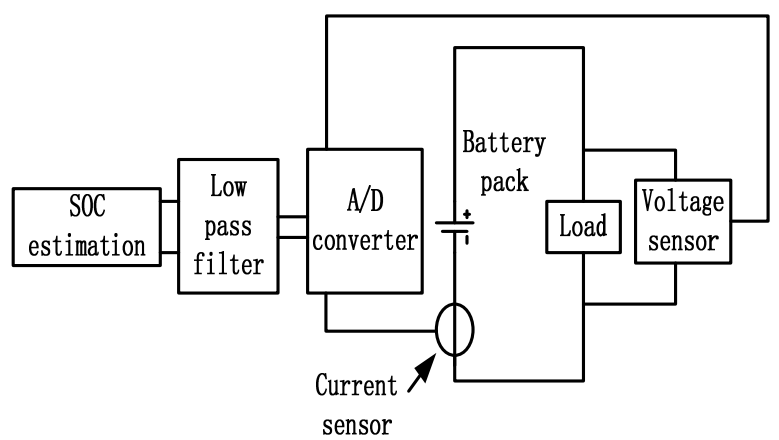

.3 The experimental system structure diagram

The Lithium ion battery used in experiment is 26650, the capacity of single battery is 2200 mah, the highest voltage is $3.65 \mathrm{~V}$, and discharge termination minimum voltage is $2.84 \mathrm{~V}$, lower than this voltage will lead to battery capacity decline seriously or even scrapped.

Lithium ion battery parameters is as follow :

$$
\begin{aligned}
& C_{c b}=11252.4 F, C_{c s}=27.31 F, R i=0.1174 \Omega \\
& R t=0.1645 \Omega, \quad k=4.38, \quad \mathrm{~d}=23.74
\end{aligned}
$$

Error and noise covariance matrix selected as the following values :

$$
\boldsymbol{P}=\left[\begin{array}{ll}
2 & 0 \\
0 & 2
\end{array}\right], \quad \boldsymbol{R}=\left[\begin{array}{cc}
0.005 & 0 \\
0 & 0.0191
\end{array}\right], \quad \boldsymbol{Q}=2
$$

B . The experimental results

In order to simulate Lithium ion battery charge and discharge state in normal use and verify the effectiveness of the SOC estimation procedure, the following charging experiments have been conducted: Under normal temperature, battery is charged from OCV $=2.8435 \mathrm{~V}$ reference state (namely SOC 0\% status) under constant current till OCV = $3.6517 \mathrm{~V}$ (namely SOC $100 \%$ status), observe the state change and error of voltage and SOC.

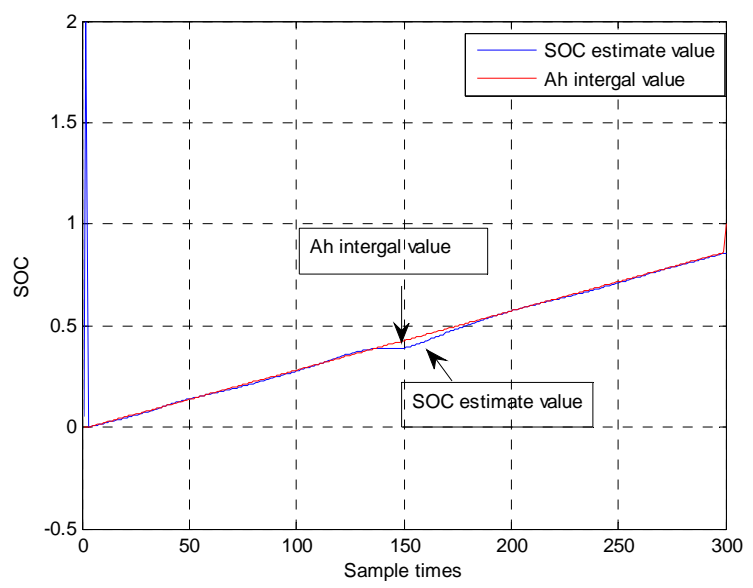

Fig.4 SOC estimation value and Ah integral value

Figure 4 is constant current charging experiment waveform diagram, SOC estimation results and expected Ah integral values are coincide basically, which explains that EKF algorithm can achieve accurate SOC estimation values.

\section{CONCLUSION}

A new method for state of charge estimation based on extended Kalman filter algorithm has been presented in this article, after obtain the relationship of Lithium ion battery SOC and OCV, firstly confirm the parameters of Lithium ion battery model, and secondly plug these parameters into state equation and observation equation, the current and voltage that measured will be used in equations, and then through the EKF equations iterative calculation and update system status constantly, an accurate estimation value can be obtained ultimately. This kind of EKF calculation algorithm has been validated through the simulation and experiment that the SOC estimation based on EKF algorithm is effective and accurate. If the difference between noise value in equations and the actual noise value are too big, estimated value is difficult to converge to a reasonable range. How to estimate the influence of noise in the algorithm is the next step research direction.

\section{REFERENCES}

[1] Gregory L.Plett. "Extended Kalman filtering for battery management systems of LiPB-based HEV battery packs" Part 1.Background,Power Sources,2004,134(2):252 261

[2] Gregory L.Plett. "Extended Kalman filtering for battery management systems of LiPB-based HEV battery packs" Part 2.Modeling and identification, Power Sources, 2004, 134(2):262 276

[3] Gregory L.Plett. "Extended Kalman filtering for battery management systems of LiPB-based HEV battery packs” Part 3.State and parameter estimation, Power Sources, 2004, 134(2):277 292

[4]Mark Verbrugge, Edward Tate. “Adaptive state of charge algorithm for nickel metal hybrid batteries including hysteresis phenomena”: Journal of Power Sources, 2004(126):236-249

[5]Mark Verbrugge, Brian Koch. "Generialized Recursive Algorithm for Adaptive Multi parameter” Regression.Journal of Electrochemical SOCiety,153(1)(2006): A187 A201

[6] Minoru Kitagawa, Hiroshi Shibuya, Jun Takehara. "Development of Battery State of Charge Indicator for Electric Vehicles”, Proc.of the 11th International Electric Vehicle Symposium(1992) 
Proceedings of the 2nd International Conference on Computer Science and Electronics Engineering (ICCSEE 2013)

[7] T.Shinpo. "Develpment of Battery Management System for Electric Vehicle”.Proc.of the 14th International Electric Vehicle Sysposium(1997), 2004 (24) (2) : 41 4 